Artigo

\title{
Resultados de Intervenções em Habilidades Sociais na Redução de Bullying Escolar: Revisão Sistemática com Metanálise
}

\author{
Jorge Luiz da Silva ${ }^{4^{*}}$ \\ Orcid.org/0000-0002-3727-8490 \\ Wanderlei Abadio de Oliveira ${ }^{1}$ \\ Orcid.org/0000-0002-3146-8197 \\ Marcela Almeida Zequinão ${ }^{5}$ \\ Orcid.org/0000-0003-3570-5425 \\ Elisângela Aparecida da Silva Lizzi ${ }^{3}$ \\ Orcid.org/0000-0001-7064-263X \\ Beatriz Oliveira Pereira ${ }^{2}$ \\ Orcid.org/0000-0003-4771-9402 \\ Marta Angélica Iossi Silva ${ }^{1}$ \\ Orcid.org/0000-0002-9967-8158

${ }^{1}$ Universidade de São Paulo, Ribeirão Preto, SP, Brasil
${ }^{2}$ Universidade do Minho, Braga, Portugal
${ }^{3}$ Universidade Tecnológica Federal do Paraná, Cornélio Procópio, PR, Brasil
${ }^{4}$ Universidade de Franca, Franca, SP, Brasil
${ }^{5}$ Universidade do Oeste de Santa Catarina, Joaçaba, SC, Brasil

\section{Resumo}

O bullying, considerado problema de saúde pública, afeta o relacionamento social, o desempenho escolar e a saúde de crianças e adolescentes. Esta revisão de literatura com metanálise objetivou verificar se intervenções em habilidades sociais reduzem a vitimização e/ou agressão por bullying. Foram consultadas as bases de dados: LILACS, PsycINFO, Scielo, SCOPUS e Web of Science. Na metanálise, empregou-se o modelo de efeitos aleatórios e o método de DerSimonian-Laird. Selecionou-se os seis estudos que atenderam aos critérios de inclusão, cuja qualidade metodológica foi avaliada pela escala de Downs e Black. Os resultados indicaram efeitos das intervenções em habilidades sociais na redução de agressão e vitimização, porém em níveis não significativos. Intervenções em habilidades sociais podem ser mais eficazes se desenvolvidas em conjunto com outras que envolvam também a variedade de situações, contextos e sujeitos implicados no bullying, como equipe escolar e família.

Palavras-chave: Bullying, habilidades sociais, intervenção, metanálise.

* Endereço para correspondência: Programa de Pós-Graduação em Promoção de Saúde, Universidade de Franca, Avenida Dr. Armando Salles Oliveira, 201, Parque Universitário, Franca, SP, Brasil 144040-600. Fone: (16) 3011-8728; Fax: (16) 3011-8728. E-mail: jorge.silva@unifran.edu.br

Projeto financiado pela Fundação de Amparo à Pesquisa do Estado de São Paulo (FAPESP). Processos 2013/22361-5 e 2015/01794-6. 


\title{
Results from Interventions Addressing Social Skills to Reduce School Bullying: A Systematic Review with Meta-Analysis
}

\begin{abstract}
Bullying, a public health problem, affects social relationships, school performance and students' health. The objective of this literature review was to verify whether interventions addressing social skills reduce victimization and/or aggression resulting from bullying. The following databases were consulted: LILACS, PsycINFO, Scielo, SCOPUS and Web of Science. The random-effects model and DerSimonian-Laird method were used. Six studies that met the inclusion criteria were selected, the methodological quality of which was assessed using the Downs and Black scale. Results indicated that interventions addressing social skills reduced bullying and victimization, however, at non-significant levels. Interventions addressing social skills may be more effective if developed together with other types of intervention which may include a wide range of situations, contexts, as well as the different individuals involved in the bullying incident, such as school staff and family.
\end{abstract}

Keywords: Bullying, social skills, intervention, meta-analysis.

\section{Resultados de Intervenciones en Habilidades Sociales Aplicadas a la Reducción del Acoso Escolar: Revisión Sistemática con Metaanálisis}

\section{Resumen}

El Bullying o acoso escolar, considerado un problema de salud pública, afecta a las relaciones sociales, al desarrollo escolar y a la salud en general de niños y adolescentes. Esta revisión sistemática de literatura con metaanálisis tiene como objetivo comprobar si las intervenciones en habilidades sociales reducen la victimización y/o la agresión en los casos de acoso escolar. Se consultaron las bases de datos LILACS, PsycINFO, Scielo, SCOPUS y Web of Science. En el metaanáisis se empleó el modelo de efectos aleatórios y el modelo de DerSimonian-Laird. Se seleccionaron los seis estudios que corresponden a los critérios de exclusión cuya calidad metodológica fue evaluada en la escala de Downs y Black. Los resultados indicaron efectos positivos en las intervenciones pero a un nivel muy poco significativo. Las intervenciones podrían ser más eficaces si se desarrollan en conjunto con otras que aporten variedad en cuanto a situaciones, contextos y sujetos implicados en el acoso escolar tales como el personal docente y la familia.

Palabras clave: Acoso escolar, habilidades sociales, metaanálisis.

Dificuldades de relacionamento entre pares na escola representam uma realidade para muitas crianças e adolescentes. Um problema grave nesse contexto se refere às situações de conflito assinaladas por violência, sendo o bullying a forma mais frequente no ambiente escolar (Olweus, 2013). Esse fenômeno representa um tipo de violência entre pares que ocorre mediante comportamentos agressivos intencionais, repetitivos e realizados em relação desigual de poder, no qual os estudantes podem participar na condição de vítima, agressor, vítima-agressora e/ou testemunha de agressões contra colegas (Silva, Oliveira, \& Longarezi, 2008).

A prevalência média de participação no bullying escolar na América do Norte e Europa, identificada em uma pesquisa transcultural desenvolvida em 28 países, com uma amostra total de 123.227 estudantes, foi de aproximadamente $40 \%$ (Due et al., 2005). No Brasil, a ocorrência do fenômeno foi mapeada pela Pesquisa Nacional de Saúde do Escolar (PeNSE) que, em sua 
segunda edição realizada no ano de 2012, identificou participação de $28 \%$ em uma amostra de 109.104 estudantes de escolas públicas e privadas de todos os estados brasileiros (Oliveira et al., 2015). A presença de situações de bullying colabora para que o ambiente escolar seja percebido pelos estudantes como local pouco seguro (Skrzypiec, Slee, Murray-Harvey, \& Pereira, 2011).

Esses dados indicam um cenário preocupante para profissionais da educação, psicologia e saúde, bem como para o desenvolvimento de políticas públicas em diferentes áreas, uma vez que, para além da elevada prevalência, as consequências negativas do fenômeno repercutem na escolaridade, desenvolvimento psicossocial e condições de saúde dos estudantes. Resumidamente, se encontram associados ao bullying: depressão, ansiedade, solidão, comportamentos infracionais, indisciplina, reprovação escolar, evasão dos estudos, uso de álcool e outras drogas, automutilação e suicídio (Benedict, Vivier, \& Gjelsvik, 2015; Silva \& Bazon, 2014; Silva, Silva, Pereira, Oliveira, \& Medeiros, 2014).

A literatura indica as habilidades sociais pouco desenvolvidas como uma das causas para o bullying, crianças ou adolescentes que apresentam essa característica se encontram mais vulneráveis a sofrerem agressões (Stan \& Beldean, 2014). Habilidades sociais representam "diferentes classes de comportamentos sociais do repertório de um indivíduo, que contribuem para a competência social, favorecendo um relacionamento saudável e produtivo com as demais pessoas" (Del Prette \& Del Prette, 2013, p. 31). Alguns exemplos de classes de habilidades sociais são as habilidades de comunicação, habilidades de civilidade, habilidades assertivas de enfrentamento, habilidades empáticas, entre outras. Já a competência social apresenta um sentido avaliativo por corresponder à habilidade dos indivíduos em utilizar seus recursos internos (pensamentos e sentimentos) em articulação com os recursos externos (aspectos sociais e culturais) para atingirem um objetivo pessoal com consequências positivas para si mesmo e para as outras pessoas (Del Prette \& Del Prette, 2013).
As vítimas de bullying apresentam características relacionadas à ausência de habilidades sociais adequadas, como o isolamento social e estratégias de enfrentamento ineficazes, como por exemplo: chorar e ignorar o agressor (Fox \& Boulton, 2003). Essas estratégias, de modo geral, indicam que as vítimas não são socialmente competentes, pois sinalizam aos agressores a ausência de condições para autodefesa, o que faz com que a violência se intensifique (Crawford \& Manassis, 2011). Portanto, a melhoria das habilidades sociais, especialmente da assertividade, representa um aspecto importante para basear intervenções destinadas à redução do bullying para as vítimas (Silva et al., 2016). Já para os agressores, não há consenso na literatura, contudo, existem indicações de que possuem dificuldades para lidarem com desafios interpessoais e que a melhoria de suas habilidades sociais, especialmente a empatia, pode reduzir as agressões que praticam (Stan \& Beldean, 2014).

Malgrado a potencialidade que as intervenções com foco em habilidades sociais apresentam como estratégia de prevenção e redução das situações de vitimização e agressão por bullying escolar, e apesar de estudos já virem apontando nessa direção, verifica-se que ainda não foram divulgadas revisões de literatura sobre a efetividade dos programas de intervenção em habilidades sociais para a redução do bullying escolar. Identificar na literatura científica as iniciativas exitosas ou pouco eficazes em relação às estratégias antibullying constitui tarefa essencial à formulação de novas propostas ou reprodução daquelas mais efetivas. Assim, o objetivo deste trabalho foi verificar se intervenções baseadas em habilidades sociais reduzem a vitimização e/ ou agressão por bullying.

\section{Método}

\section{Tipo de Estudo}

Para se atender ao objetivo proposto realizou-se uma revisão sistemática da literatura com metanálise. A revisão sistemática consiste em uma síntese rigorosa de estudos sobre um mesmo tema, na qual se busca limitar possíveis vieses presentes nos processos de seleção e aná- 
lise dos trabalhos. Seus princípios gerais são: a variedade de locais de busca dos estudos a serem analisados, a elaboração prévia de critérios de inclusão e exclusão e a avaliação da qualidade metodológica dos estudos. Neste estudo foi integrada a metanálise na revisão sistemática, por ela representar uma técnica estatística adequada para combinar resultados de diferentes investigações (Galvão \& Pereira, 2014).

\section{Bases de Dados e Busca Bibliográfica}

O levantamento das produções referentes às temáticas investigadas ocorreu em outubro de 2015 em quatro bases de dados internacionais (LILACS, PsycINFO, SCOPUS e Web of Science) e uma biblioteca eletrônica nacional (SciELO). Como estratégia de busca realizou-se o cruzamento dos termos: Bullying AND Social Skills; Bullying AND Social Skills Training e os seus correlatos em português. A busca foi norteada pela pergunta: "Qual o resultado apresentado por intervenções baseadas em habilidades sociais e realizadas na escola na redução do bullying escolar?". Essa questão de pesquisa foi elaborada com base na estratégia PICO (Patient or Problem, Intervention, Control or Comparasion, Outcomes; Santos, Pimenta \& Nobre, 2007).

\section{Critérios de Inclusão e Exclusão}

Optou-se pela inclusão somente de artigos, excluindo da análise outros tipos de publicação como editoriais, livros, capítulos de livro, teses e dissertações. Elegeu-se também apenas os trabalhos com foco específico no bullying e não em outros tipos de violência, desconsiderando, por exemplo, aqueles direcionados exclusivamente à homofobia. Foram incluídas somente as intervenções em habilidades sociais, com delineamento experimental ou quase experimental, e cujos resultados tenham sido avaliados em comparação a estudantes que não receberam a intervenção (grupo controle). Assim, não foram selecionados os estudos com avaliação antes e depois sem grupo controle, por não possibilitarem a comparação com outros sujeitos com características semelhantes, aspecto limitante da interpretação dos resultados obtidos (Ttofi \&
Farrington, 2011). Não houve recorte temporal quanto à data de publicação, porém somente foram incluídas as produções em espanhol, inglês e português.

\section{Extração de Dados}

Os estudos foram selecionados inicialmente mediante a leitura de seus respectivos títulos e resumos, tomando por base os critérios de inclusão e exclusão. Posteriormente, os textos completos dos artigos selecionados foram analisados. Para tanto, foi elaborado um instrumento próprio para a extração dos seguintes dados: autores, ano de publicação, país de desenvolvimento do estudo, número amostral (grupo de intervenção e grupo controle), idade dos sujeitos, características da intervenção, principais resultados e limitações.

\section{Avaliação de Qualidade Metodológica}

A qualidade metodológica dos trabalhos foi avaliada através da escala de Downs e Black (1998), desenvolvida para avaliar estudos de intervenção. Trata-se de um instrumento composto por 27 questões divididas em cinco dimensões: descrição do estudo (objetivo, desfecho, critérios de inclusão, tipo de intervenção, fatores de confusão, variabilidade dos resultados, efeitos adversos da intervenção, perda amostral e probabilidade estatística), validade externa (representatividade da amostra e adequação do local de coleta dos dados), validade interna (procedimentos de cegamento, adequação da análise de dados, adesão dos participantes e acurácia nas medidas), viés de seleção (recrutamento de sujeitos, perdas amostrais, randomização e ajuste para fatores de confusão) e poder do estudo. Cada questão é pontuada com o valor de 1 ponto se atende ao critério por ela analisado, de modo que a pontuação máxima a ser obtida com o instrumento é de 27 pontos. Os resultados mais próximos a esse valor representavam melhor qualidade do estudo.

\section{Análise Estatística}

Para a realização da metanálise, utilizou-se o modelo de efeitos aleatórios e o método de DerSimonian-Laird, pressupondo-se que o efeito 
de interesse é o mesmo em todos os trabalhos e que as diferenças observadas entre eles são devidas apenas a erros amostrais (variabilidade dentro dos estudos). Para calcular as diferença entre as médias dos grupos experimental e controle antes e depois das intervenções em habilidades sociais, contou-se com o auxílio do pacote metafor (Viechtbauer, 2010) do software livre $R$ (versão 3.1.3). Os resultados foram apresentados por meio de gráficos Forest Plot. A avaliação da heterogeneidade entre os estudos e sua magnitude foi realizada mediante os testes Q de Cochran e o índice $\mathrm{I}^{2}$. As porcentagens de referência para a heterogeneidade, considerando-se o índice $\mathrm{I}^{2}$, foram: próximas de $25 \%$ (baixa), próximas de $50 \%$ (moderada) e próximas de $75 \%$ (alta; Higgins \& Thompson, 2002). Considerou-se um nível de significância de 5\%.

\section{Resultados}

A busca bibliográfica localizou 613 publicações, dentre as quais foram selecionados seis artigos que atenderem aos critérios de inclusão (Figura 1).

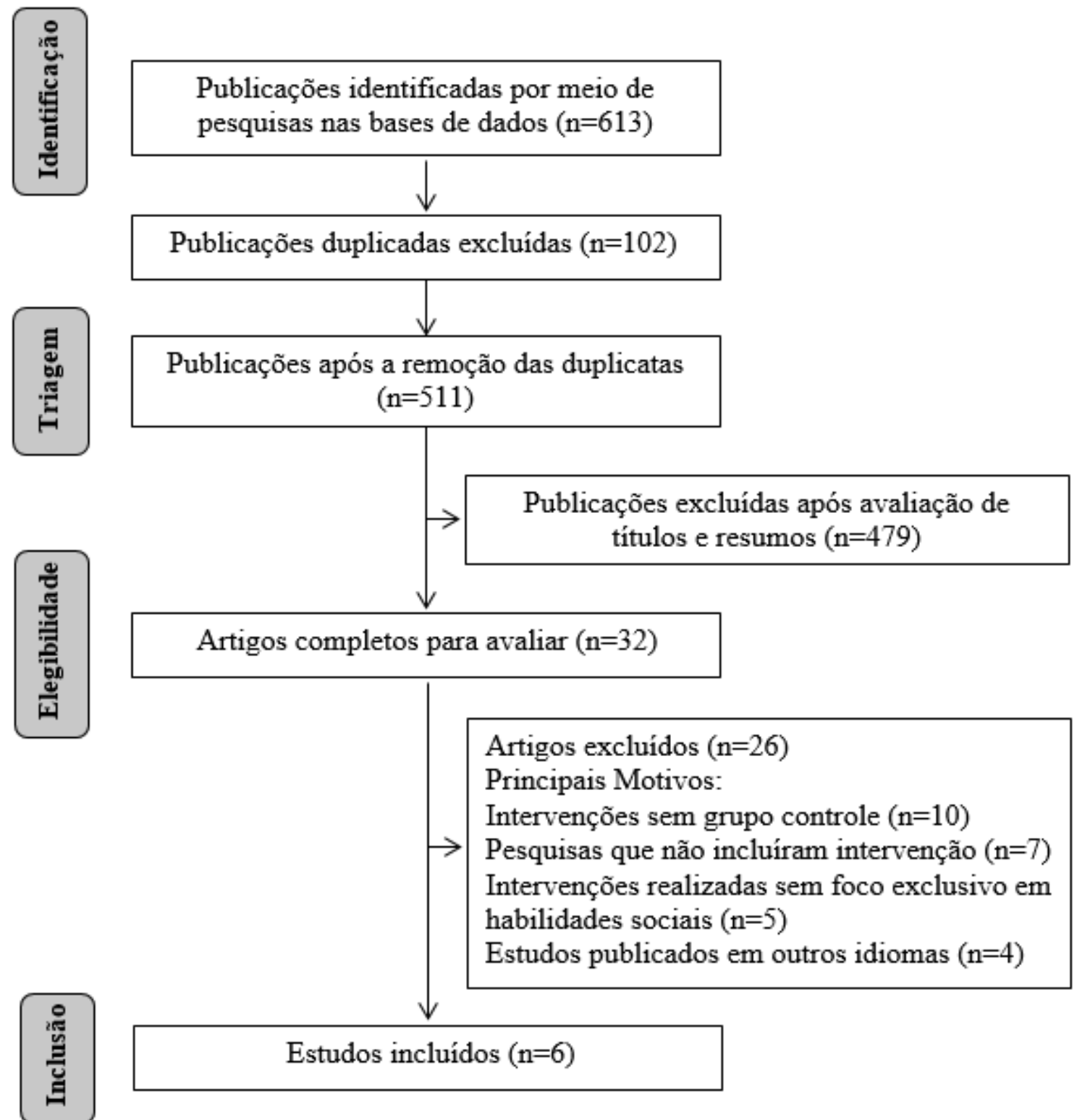

Figura 1. Fluxograma do processo de seleção dos estudos. 
Em relação aos seis artigos analisados (Tabela 1), identifica-se que o mais antigo foi publicado no ano de 2003 e o mais recente em 2014. Todos foram divulgados na língua inglesa, porém realizados em realidades socioculturais distintas: Austrália, Estados Unidos, Inglaterra, Itália, Japão e Romênia. O tamanho das amostras investigadas variou entre 28 e 381 participantes divididos em grupos de intervenção e grupos controle. Todas as intervenções foram realizadas com participantes do sexo masculino e feminino. Dois estudos ocorreram exclusivamente com crianças, dois somente com adolescentes e dois abrangeram esses dois públicos, por incluírem uma faixa etária maior entre 8 e 16 anos. Metade das intervenções se desenvolveu com estudantes em condição de vítimas, agressores e não envolvidos no bullying, duas se direcionaram unicamente a vítimas e uma somente aos agressores. $\mathrm{Na}$ sequência serão apresentados os principais resultados de cada estudo.

Tabela 1

Características dos Estudos Selecionados para a Revisão Sistemática e Metanálise

\begin{tabular}{|c|c|c|c|c|c|c|}
\hline Autoria & Ano & País & Amostra & Idade & Desenho do estudo & Objetivou reduzir \\
\hline $\begin{array}{l}\text { Ando, Asakura, Ando, } \\
\text { \& Simons-Morton }\end{array}$ & 2007 & Japão & 104 & $12-13$ & Quase Experimental & Agressão \\
\hline Baldry \& Farrington & 2004 & Itália & 237 & $10-16$ & Experimental & $\begin{array}{c}\text { Agressão } \\
\text { e vitimização }\end{array}$ \\
\hline Berry \& Hunt & 2009 & Austrália & 46 & $12-15$ & Experimental & Vitimização \\
\hline Fox \& Boulton & 2003 & Inglaterra & 28 & $9-11$ & Quase Experimental & Vitimização \\
\hline DeRosier & 2004 & $\begin{array}{l}\text { Estados } \\
\text { Unidos }\end{array}$ & 381 & $8-11$ & Experimental & $\begin{array}{c}\text { Agressão } \\
\text { e vitimização }\end{array}$ \\
\hline Stan \& Beldean & 2014 & Romênia & 231 & $10-14$ & Quase Experimental & $\begin{array}{c}\text { Agressão } \\
\text { e vitimização }\end{array}$ \\
\hline
\end{tabular}

Ando et al. (2007) avaliaram um programa de treinamento de habilidades sociais estruturado em quatro sessões, sendo uma por semana, destinadas à resolução de problemas, gerenciamento de estresse e comunicação. Participaram 104 estudantes japoneses (vítimas, agressores e não envolvidos) do sétimo ano de uma escola da cidade de Tóquio, distribuídos em grupos de intervenção $(n=52)$ e controle $(n=52)$. Dentre outros objetivos, o programa visava diminuir o bullying. Os resultados indicaram reduções em agressão, o que também ocorreu no grupo controle, sem significância estatística a favor do treinamento de habilidades sociais.

Objetivando melhorar as habilidades sociais e o entendimento das consequências negativas da violência e do bullying, o programa desenvolvido por Baldry e Farrington (2004) utilizou-se de uma amostra de 237 crianças e adolescentes de três escolas italianas da cidade de Roma. Os es- tudantes (vítimas, agressores e não envolvidos), com idade entre 10 e 16 anos, foram distribuídos aleatoriamente entre grupos de intervenção $(n=131)$ e grupos controle $(n=106)$. A intervenção ocorreu por um período de três semanas com encontros semanais com duração de três horas cada. Os participantes também receberam um livreto com os conteúdos trabalhados nos encontros. Os resultados demostraram que os efeitos positivos da intervenção aumentavam com a idade, resultando em diminuição estatisticamente significativa de vitimização e agressão $(p<0,05)$ somente para os estudantes mais velhos. Para os estudantes mais jovens, em alguns casos, ocorreu aumento da vitimização para o grupo experimental.

A intervenção desenvolvida por Berry e Hunt (2009) focou-se em características pessoais que potencialmente aumentam a vulnerabilidade dos estudantes que sofrem bullying (vítimas) 
tais como: ansiedade, baixa autoestima e estratégias de enfrentamento inadequadas. As atividades desenvolvidas em oito sessões semanais com duração de uma hora cada visavam reforçar competências pessoais, aumentar a utilização de estratégias de enfrentamento assertivas em situações de bullying e reduzir a ansiedade das vítimas. A amostra do estudo foi recrutada em sete escolas da cidade de Sydney na Austrália e constitui-se de 46 estudantes do sexo masculino, estudantes do sétimo ao décimo ano escolar que sofriam bullying e relatavam sintomas de ansiedade. Os estudantes foram distribuídos aleatoriamente no grupo de intervenção $(n=22)$ e controle $(n=24)$. No tocante à vitimização, os resultados apresentaram redução significativa a favor do grupo de intervenção $(p<0,001)$.

Fox e Boulton (2003) elaboraram um programa de treinamento de habilidades sociais denominado Social Skills Training (SST) que foi desenvolvido em quatro escolas inglesas com 15 estudantes com média de idade de nove anos e seis meses. Todos os participantes eram vítimas de bullying e o programa objetivou melhorar suas habilidades sociais e assim reduzir a vulnerabilidade que apresentavam para sofrerem agressões. As habilidades enfocadas nos oito encontros semanais realizados com duração de uma hora cada foram: solução de problemas, pensamento positivo, relaxamento, linguagem corporal, fazer amizade e lidar com agressor. Os resultados atinentes à vitimização não revelaram diferenças estatisticamente significativas após a intervenção, em comparação ao grupo controle $(n=13)$.

DeRosier (2004) avaliou os efeitos de um programa de treinamento de habilidades sociais desenvolvido com crianças (vítimas, agressores e não envolvidos) do terceiro ano escolar identificadas previamente como sendo ansiosas, rejeitadas por pares ou agressivas. Foram realizadas oito sessões grupais com duração de uma hora cada, durante oito semanas, com atividades direcionadas a habilidades sociais, regulação emocional e identificação de pensamentos negativos. As crianças foram distribuídas aleatoriamente entre os grupos de intervenção $(n=187)$ e controle $(n=194)$. Participaram do estudo 11 esco- las do condado de Wake na Carolina do Norte, Estados Unidos. Em relação ao bullying, especificamente, a intervenção diminuiu a ocorrência de agressões em nível significativo $(p<0,05)$ somente para crianças identificadas inicialmente como agressivas.

O estudo de Stan e Beldean (2014) objetivou verificar se o desenvolvimento de habilidades sociais dos estudantes reduz a ocorrência de bullying. Para tanto, desenvolveram uma intervenção focada em identificação de pensamentos negativos, regulação emocional e assertividade. Participaram 231 estudantes (vítimas, agressores e não envolvidos) do quinto ao oitavo anos de duas escolas da cidade de Cluj-Napoca, Romênia, sendo 117 pertencentes ao grupo experimental e 114 ao grupo controle. As sessões foram realizadas semanalmente totalizando 18 horas. Os resultados indicaram redução da vitimização e da agressão para os grupos participantes da intervenção, porém em níveis não significativos quando comparados aos resultados dos grupos controle.

No tocante à avaliação da qualidade metodológica estudos selecionados, a escala de Downs e Black (1998) indicou uma mediana de 22 pontos, com amplitude de 19-24 pontos, tomando-se por referência a pontuação máxima que poderia ser obtida de 27 pontos (Tabela 2). As fragilidades mais recorrentes foram: não descrição dos fatores de confusão, desconsideração de efeitos adversos da intervenção, ausência de descrição das perdas amostrais, não cegamento dos participantes e dos avaliadores, falta de randomização, ausência de ajustes para fatores de confusão e não apresentação do poder do estudo.

Para avaliar o efeito das intervenções em habilidades sociais na redução do bullying, os estudos resultantes da revisão sistemática foram analisados na metanálise, por meio do modelo de efeitos aleatórios e o método de DerSimonian-Laird. Os Forest Plot inseridos nas Figuras $2 \mathrm{e}$ 3 mostram a análise do efeito das intervenções em relação à agressão e vitimização, respectivamente, em comparação com outros estudantes que não participaram da intervenção (grupo controle). As linhas horizontais representam a 
Tabela 2

Critérios para Avaliação dos Estudos Selecionados, com Base em Downs e Black (1998)

Critérios $(n=6)$

Acertos (\%)

Descrição do estudo

Apresenta hipóteses/objetivos

100

Desfechos bem definidos 100

Critérios de inclusão bem definidos 100

Tipo de intervenção bem definido 100

Descrição clara dos fatores de confusão

Principais achados bem descritos 100

Apresenta estimativas de variabilidade dos resultados

Relata efeitos adversos da intervenção

Descreve as perdas

Apresenta intervalo de $95 \%$ de confiança ou valor de $p$

Validade externa

Sujeitos chamados para o estudo representam a população

Sujeitos que participaram do estudo representam a população

Lugares/Instalações representativos

Validade interna

Cegamento dos participantes

Cegamento dos avaliadores

Resultados baseados em dragagem de dados 100

Ajuste para tempo de acompanhamento 100

Testes estatísticos apropriados 100

Adesão confiável às intervenções 100

Medidas acuradas dos desfechos

Viés de seleção

Grupos intervenção/controle recrutados da mesma população 100

Grupos intervenção/controle recrutados ao mesmo tempo

Foi realizada randomização

Randomização completa e irrevogável

Teve ajuste para fatores de confusão

Perdas foram consideradas

diferença média de cada estudo entre os grupos experimental e controle após a intervenção, com os respectivos intervalos de confiança (IC95\%). Os dados que se localizam à direita da linha ver- tical indicam que a intervenção foi favorável à redução do bullying para a intervenção, estando o efeito combinado de todos os estudos representado pelo losango na linha denominada total. 


\begin{tabular}{|c|c|c|c|c|c|c|c|c|c|}
\hline \multirow{2}{*}{ Estudos } & \multicolumn{3}{|c|}{ Intervenção } & \multicolumn{3}{|c|}{ Controle } & \multirow{2}{*}{$\begin{array}{c}\text { Peso do } \\
\text { estudo (\%) }\end{array}$} & \multirow{2}{*}{$\begin{array}{c}\text { Diferença Média } \\
\text { (IC95\%) }\end{array}$} & \multirow{2}{*}{$\begin{array}{c}\text { Diferença Média } \\
\text { (IC95\%) }\end{array}$} \\
\hline & M & $\mathrm{DP}$ & $\mathrm{n}$ & $\mathrm{M}$ & DP & $\mathrm{n}$ & & & \\
\hline Ando et al. (2007) & 0,96 & 1,58 & 52 & 0,85 & 1,26 & 52 & 26,1 & $0,11[-0,44,0,66]$ & \\
\hline Baldry \& Farrington $(2004)^{1}$ & 2,69 & 3,31 & 58 & 1,57 & 2,20 & 72 & 17,7 & $1,12[0,13,2,11]$ & \\
\hline Baldry \& Farrington $(2004)^{2}$ & 2,31 & 3,07 & 63 & 3,39 & 3,99 & 36 & 10,9 & $-1,08[-2,59,0,43]$ & - \\
\hline DeRosier (2004) & 0,15 & 1,22 & 187 & 0,07 & 1,13 & 194 & 31,6 & $0,08[-0,16,0,32]$ & - \\
\hline Stan \& Beldean (2014) & 5,38 & 4,82 & 115 & 7,08 & 4,99 & 117 & 13,7 & $-1,70[-2,96,-0,44]$ & 一 \\
\hline Total & & & 475 & & & 471 & 100 & $-0,10[-0,71,0,51]$ & - \\
\hline
\end{tabular}

Heterogeneidade: $Q=14,11, \mathrm{df}=4(\mathrm{p}=0,007) ; \mathrm{I}^{2}=71,75 \%$

Teste para efeito global: $Z=-0,3193(p=0,74)$

Figura 2. Forest Plot para as diferenças médias na redução de agressão no bullying após as intervenções em habilidades sociais.

Os resultados apresentados na Figura 2 demonstram efeitos estatisticamente não significativos das intervenções na redução de episódios de agressão, com diferença média combinada: $-0,10$ (IC95\%: -0,71, 0,51). Houve heterogeneidade entre os estudos, com variabilidade alta $\left(\mathrm{I}^{2}=71,75 \%\right)$, justificando a escolha do teste de DerSimonian-Laird.

$\mathrm{Na}$ Figura 3, a análise do teste $\mathrm{Q}$ de Cochran para a heterogeneidade dos resultados das intervenções referentes à vitimização revelou diferença média combinada: -0,19 (IC95\% -0,89, 0,52), com 5 graus de liberdade, sem significância estatística. A existência de heterogeneidade foi baixa entre os estudos $\left(\mathrm{I}^{2}=45,52 \%\right)$. Na comparação em conjunto dos estudos analisados, as intervenções em habilidades sociais não apresentaram para as vítimas reduções do bullying em níveis significativos.

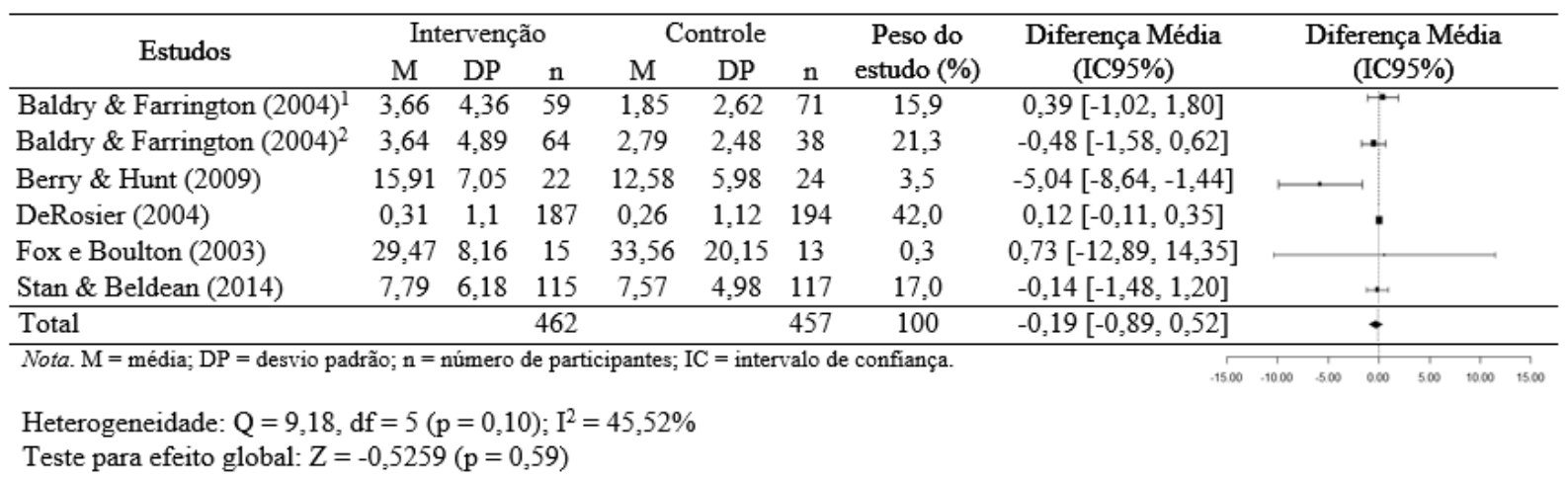

Figura 3. Forest Plot para as diferenças médias na redução de vitimização por bullying após as intervenções em habilidades sociais.

Na Figura 3, a análise do teste Q de Cochran para a heterogeneidade dos resultados das intervenções referentes à vitimização revelou diferença média combinada: $-0,19$ (IC95\% $-0,89$, 0,52), com 5 graus de liberdade, sem significância estatística. A existência de heterogeneidade foi baixa entre os estudos $\left(\mathrm{I}^{2}=45,52 \%\right)$. Na comparação em conjunto dos estudos analisados, as intervenções em habilidades sociais não apresentaram para as vítimas reduções do bullying em níveis significativos.

\section{Discussão}

A presente investigação objetivou verificar se intervenções em habilidades sociais reduzem a vitimização e/ou agressão por bullying. A pequena quantidade de estudos com delineamento experimental ou quase experimental $(n=6)$ identificada nas bases de dados consultadas denota que as intervenções em habilidades sociais têm sido pouco desenvolvidas na prevenção ou redução do bullying com delineamentos meto- 
dológicos mais rigorosos. Talvez isso se deva aos resultados divergentes obtidos por outras pesquisas com delineamento semelhante ou às indicações da literatura de que intervenções denominadas de "toda a escola", envolvendo a participação de estudantes, famílias, professores e demais membros da comunidade escolar, logram resultados mais promissores, por abrangerem de modo mais completo a variedade de situações, contextos e sujeitos implicados no bullying (Olweus, 2013).

Assim, intervenções focais direcionadas a sujeitos específicos como os estudantes, público preferencial das intervenções em habilidades sociais, são pouco desenvolvidas por conta de apresentarem resultados menos positivos (Ttofi \& Farrington, 2011). O caráter recente dos estudos recuperados nesta revisão talvez possa também estar a isso relacionado, uma vez que as intervenções denominadas de toda a escola, como o Olweus Bullying Prevention Program (OBPP), são desenvolvidas desde a década de 1980 (Olweus, 2013) e o estudo mais antigo sobre intervenção em habilidades sociais localizado nesta revisão foi publicado em 2003.

As investigações selecionadas demonstraram possuir qualidade metodológica suficiente, todas acima de $70 \%$ no atendimento ao total 27 características avaliadas, o que garante maior confiabilidade nos resultados obtidos, apesar do fato de alguns estudos não terem considerado variáveis de confusão que podem ter interferido em seus resultados, não terem descrito as perdas amostrais ou feito randomização, assim como a maioria não ter apresentado o poder do estudo.

Outro aspecto que merece destaque é a variedade de realidades socioculturais em que as intervenções foram desenvolvidas (países diferentes), considerando-se que o sucesso obtido por alguma intervenção em determinado contexto não é garantia de resultados semelhantes em outro, em função das diferenças existentes (Napolitano, Espelage, Vaillancourt, \& Hymel, 2010). Um exemplo é o programa OBPP de Olweus que apresenta resultados divergentes a depender do país em que é desenvolvido, com maiores níveis de sucesso em países europeus e resultados quase nulos nos Estados Unidos, país no qual a maioria das intervenções sobre bullying alcança resultados menores (Ttofi \& Farrington, 2011).

De modo geral, uma análise de todos os estudos selecionados evidencia que a efetividade das intervenções variou entre situações de ausência de diminuição significativa da quantidade de bullying (vitimização ou agressão; Ando et al., 2007; Fox \& Bouton, 2003), a outras em que ele diminuiu significativamente (Baldry \& Farrington, 2004; Berry \& Hunt, 2009; DeRosier, 2004; Stan \& Beldean, 2014).

A metanálise sobre os efeitos das intervenções em habilidades sociais para a redução da vitimização por bullying não logrou diferenças significativas, embora tenha havido diminuições em alguns estudos. A literatura indica que nas situações de vitimização deveriam ocorrer as maiores reduções por intervenções que melhorassem as habilidades e competência social dos estudantes, uma vez que se diminuiria os déficits de habilidades sociais, ausência de assertividade e comportamentos direcionados ao isolamento social (Fox \& Boulton, 2003; Stan \& Beldean, 2014).

Essa aparente contradição, ausência de diminuição significativa de vitimização, pode talvez ser esclarecida ao se considerar o bullying enquanto fenômeno de grupo e também influenciado por características dos contextos em que ocorre, tais como da sala de aula ou da escola em sua totalidade, o que restringe as possibilidades reais de uma criança ou adolescente poderem superar sozinhos um comportamento amplamente aceito ou promovido pelo coletivo de pares (Wölfer \& Scheithauer, 2014). Assim, mesmo as respostas assertivas perante as agressões sofridas podem não ser efetivas em um contexto em que a violência é considerada normativa.

Em termos de metodologia das intervenções em habilidades sociais, a dificuldade em se generalizar as habilidades aprendidas para situações cotidianas reais é um aspecto reconhecido (Silva et al., 2016). Como possibilidade de superação desta dificuldade, tem sido sugerida a utilização de mais técnicas de roleplay para que os alunos 
experimentem na intervenção uma maior proximidade com as situações reais por eles vivenciadas no contexto escolar (Leadbeater \& Hoglund, 2006), o que nem sempre é fácil de ocorrer se as agressões forem naturalizadas no grupo de pares. Para além dessa consideração, o agrupamento de sujeitos com características semelhantes (somente vítimas ou agressores) pode interferir negativamente no desenvolvimento das atividades, prejudicando os resultados da intervenção.

De todo modo, as diminuições na vitimização, embora modestas, precisam ser valorizadas e sinalizam a potencialidade de sucesso desta modalidade interventiva em relação ao bullying. Um passo importante a ser tomado consiste no aprimoramento das intervenções, com vistas a se identificar com maior precisão se a pequena redução ocorre devido a problemas metodológicos ou de planejamento das intervenções ou pelos motivos apresentados acima. Conforme mencionado anteriormente, mesmo intervenções mais abrangentes como as denominadas de "toda a escola" em determinados contextos apresentam resultados divergentes. Pesquisas futuras podem ajudar a esclarecer estes apontamentos.

O resultado da metanálise referente ao efeito das intervenções na diminuição específica de agressões também não foi significativo para os grupos experimentais em comparação aos grupos controles. Resultado convergente ao apresentado por outra metanálise desenvolvida por Ttofi e Farrington (2011), a qual avaliou a efetividade de diferentes modelos interventivos na redução do bullying e que, em relação àqueles direcionados às habilidades sociais, não identificou diminuição significativa nos episódios de agressão.

Assim, a prática de agressões pode não se encontrar atrelada a problemas de desenvolvimento das habilidades sociais. Esse resultado coaduna com alguns estudos que os identificam os agressores como não possuindo déficits nas habilidades sociais (Napolitano et al., 2010; Thunfors \& Cornell, 2008). Destaca-se, contudo, que a literatura sobre as habilidades sociais dos agressores apresenta resultados divergentes. $\mathrm{O}$ que também ocorre em outras investigações com delineamentos não experimentais, nos quais se identifica reduções de até 50\% nas agressões ou a ausência de efeitos significativos (Kõiv, 2012).

Embora a pequena quantidade de estudos interventivos realizados sobre a temática represente um fator limitante a uma identificação mais precisa dos efeitos exercidos sobre a agressão no bullying, quando analisados individualmente, algumas intervenções apresentaram resultados significativos. A redução por eles apresentada sobre a agressão pode ser parcialmente explicada pela opção de se incluir nas intervenções grupos de estudantes com estilos variados de comportamento em relação ao bullying, pois pode ter evitado que os agressores, que praticam mais agressões, reforçassem os comportamentos agressivos uns dos outros, bem como oferecido a oportunidade de maior contato com colegas que podem ter constituído modelo de conduta prossocial (Napolitano et al., 2010). Dentre os estudos analisados, os três que individualmente obtiveram reduções de agressividade em nível significativo (Baldry \& Farrington, 2004; DeRosier, 2004; Stan \& Beldean, 2014) adotaram um modelo misto de composição dos grupos de intervenção.

Outra possibilidade de explicação é que as habilidades sociais aprendidas podem ter propiciado a manifestação de respostas não agressivas nas relações sociais dos estudantes, especialmente dos agressores, de modo a aumentar-lhes a aceitação pelos pares (Leadbeater \& Hoglund, 2006). Resultado semelhante ocorreu no estudo não experimental de Murrieta, Ruvalcaba, Caballo e Lorenzo (2014), no qual houve redução significativa de agressão direta $(p<0,01)$ e agressão relacional $(p<0,05)$ para o grupo de estudantes agressores.

Outro dado que, no conjunto da análise dos estudos revisados, chama a atenção é que as intervenções obtiveram maiores efeitos para os estudantes mais velhos. Entretanto, a maioria delas focalizou amostras formadas por participantes com idade acima de 10 anos, o que restringe a interpretação deste resultado, pois pode estar enviesado pela quantidade de estudos focados em faixas etárias maiores. Apesar deste limite, uma 
possível explicação incide no fato de os alunos mais velhos possuírem habilidades cognitivas mais desenvolvidas, o que lhes garante maior probabilidade de compreenderem, aprenderem e colocarem em prática as habilidades sociais, bem como avaliarem a natureza prejudicial do bullying. Ou também pela probabilidade deles, enquanto vítimas, possuírem maior desenvolvimento físico ou tomarem decisões mais racionais, o que talvez os tornem mais hábeis para se autodefenderem, lidando mais eficazmente com as agressões nas relações com seus pares (Baldry \& Farrington, 2004).

Uma limitação identificada nos estudos é a ausência de avaliação de seguimento (follow up), considerando-se que algumas mudanças comportamentais demandam maior tempo para se efetivarem. Assim, a melhoria das habilidades pode impactar em outros aspectos da vida dos estudantes e relacionamento social até que efetivamente repercuta nas situações de violência por eles vivenciadas ou praticadas. Assim, outras dimensões da vida dos estudantes diretamente conectadas às habilidades sociais como, por exemplo, a amplitude da rede de pares, podem ao longo do tempo impactar positivamente nas situações de bullying, resultando em menores índices de violência entre os estudantes (Fox \& Boulton, 2003).

Outra limitação das produções analisadas é a falta de diferenciação entre os participantes referente à sua condição no bullying (vítima, agressor ou testemunha). Tão importante quanto identificar reduções significativas na vitimização ou agressão, é também localizar a mobilidade das crianças e adolescentes entre as diferentes condições, de modo a se verificar quantos deixaram de ser agressor ou vítima. São informações relevantes, uma vez que a quantidade de vítimas pode diminuir significativamente e não ser acompanhada por uma redução significativa na quantidade de vitimização, pois apesar de estarem em menor número, podem sofrer agressões em maior intensidade. O mesmo se aplica à quantidade de agressão. Pesquisas futuras podem se dedicar a preencherem essa lacuna.

O pequeno tamanho das amostras dos estudos impossibilita, ainda, a generalização dos seus resultados, pois nenhum se desenvolveu com amostra representativa e outros foram estudos piloto. Investigações com amostras maiores são necessárias e poderiam ser desenvolvidas no Brasil, uma vez que nenhuma pesquisa nacional foi identificada nas bases de dados consultadas. Outra limitação ocorre em relação à análise agrupada dos efeitos das intervenções para meninos e meninas. Como a prática de agressões e as respostas à intimidação são igualmente moduladas por questões relacionadas ao sexo dos envolvidos (Sentse, Kretschmer, \& Salmivalli, 2015), depreende-se a necessidade do desenvolvimento de uma compreensão matizada dos efeitos das intervenções em habilidades sociais para meninos e meninas em relação ao bullying. Pesquisas futuras também podem avançar nessa direção, incorporando as diferenças entre os sexos na análise de seus resultados.

\section{Considerações Finais}

As intervenções analisadas nesta revisão apresentaram resultados divergentes no tocante à redução de bullying, tendo apenas algumas delas produzido individualmente efeitos positivos e significativos. Contudo, na metanálise não ocorreu significância estatística. Identifica-se como uma limitação desta revisão a ocorrência de heterogeneidade alta entre os estudos relacionados à agressão, apesar de se ter analisado somente investigações com o mesmo delineamento metodológico e utilizado para a metanálise um modelo de efeitos aleatórios, análise do efeito aleatório de DerSimonian-Laird, que considera a variação dentro de cada trabalho, bem como a diferença entre os estudos na análise do efeito de interesse. Assim, é importante destacar que esses resultados, especificamente, devem ser interpretados com cautela, evitando-se generalizações. Considerando-se a relevância da temática, reforça-se a necessidade de realização de outros estudos, com delineamentos experimentais ou quase experimentais e amostras maiores, especialmente na realidade nacional, com vistas à superação das limitações identificadas e à obtenção de resultados mais robustos. 


\section{Referências}

Ando, M., Asakura, T., Ando, S., \& Simons-Morton, B. (2007). A psychoeducational program to prevent aggressive behavior among Japanese early adolescentes. Health Education \& Behavior, 34(5), 765-776. doi:10.1177/1090198106291965

Baldry, A. C., \& Farrington, D. P. (2004). Evaluation of an intervention program for the reduction of bullying and victimization in schools. Aggressive Behavior, 30(1), 1-15. doi:10.1002/ ab.20000

Benedict, F. T., Vivier, P. M., \& Gjelsvik, A. (2015). Mental health and bullying in the United States among children aged 6 to 17 years. Journal of Interpersonal Violence, 30(5), 782-795. doi:10.1177/0886260514536279

Berry, K., \& Hunt, C. J. (2009). Evaluation of an intervention program for anxious adolescent boys who are bullied at school. Journal of Adolescent Health, 45(4), 376-382. doi:10.1016/j.jadohealth.2009.04.023

Crawford, A. M., \& Manassis, K. (2011). Anxiety, social skills, friendship quality, and peer victimization: An integrated model. Journal of Anxiety Disorders, 25(7), 924-931. doi:10.1016/j.janxdis.2011.05.005

Del Prette, Z. A. P., \& Del Prette, A. (2013). Psicologia das habilidades sociais na infância: Teoria e prática. Petrópolis, RJ: Vozes.

DeRosier, M. E. (2004). Building relationships and combating bullying: Effectiveness of a schoolbased social skills group intervention. Journal of Clinical Child \& Adolescent Psychology, 33(1), 196-201. doi:10.1207/S15374424JCCP3301_18

Downs, S. H., \& Black, N. (1998). The feasibility of creating a checklist for the assessment of the methodological quality both of randomised and non-randomised studies of health care interventions. Journal of Epidemiology and Community Health, 52(6), 377-384.

Due, P., Holstein, B. E., Lynch, J., Diderichsen, F., Gabhain, S. N., Scheidt, P., \& Currie, C. (2005). Bullying and symptoms among school-aged children: International comparative cross sectional study in 28 countries. The European Journal of Public Health, 15(2), 128-132. doi:10.1093/eurpub/cki105

Fox, C., \& Boulton, M. (2003). Evaluating the effectiveness of a Social Skills Training
(SST) programme for victims of bullying. Educational Research, 45(3), 231-247. doi:10.1080/0013188032000137238

Galvão, T. F., \& Pereira, M. G. (2014). Revisões sistemáticas da literatura: Passos para sua elaboração. Epidemiologia e Serviços de Saúde, 23(1), 183184. doi:10.5123/S1679-49742014000100018

Higgins, J. P. T., \& Thompson, S. G. (2002). Quantifying heterogeneity in a metaanalysis. Statistics in Medicine, 21, 1539-1558. doi:10.1002/sim.1186

Kõiv, K. (2012). Social Skills Training as a mean of improving intervention for bullies and victims. Procedia - Social and Behavioral Sciences, 45, 239-246. doi:10.1016/j.sbspro.2012.06.560

Leadbeater, B., \& Hoglund, W. (2006). Changing the social contexts of peer victimization. Journal of the Canadian Academy of Child and Adolescent, 15(1), 21-26.

Murrieta, P., Ruvalcaba, N. A., Caballo, V. E., \& Lorenzo, M. (2014). Cambios en la percepción de la violencia y el comportamiento agresivo entre niños a partir de un programa de habilidades socioemocionales. Psicología Conductual, 22(3), 569-584.

Napolitano, S. M. S., Espelage, D. L., Vaillancourt, T., \& Hymel, S. (2010). What can be done about school bullying? Linking research to educational practice. Educational Researcher, 39(1), 38-47. doi:10.3102/0013189X09357622

Oliveira, W. A., Silva, M. A. I., Mello, F. C. M., Porto, D. L., Yoshinaga, A. C. M., \& Malta, D. C. (2015). Causas do bullying: Resultados da Pesquisa Nacional de Saúde do Escolar. Revista Latino-Americana de Enfermagem, 23(2). doi:10.1590/0104-1169.0022.2552

Olweus, D. (2013). School bullying: Development and some important challenges. Annual Review of Clinical Psychology, 9, 751-780. doi:10.1146/ annurev-clinpsy-050212-185516

Santos, C. M. C., Pimenta, C. A. M., \& Nobre, M. R. C. (2007). A estratégia PICO para a construção da pergunta de pesquisa e busca de evidências. Revista Latino-Americana de Enfermagem, 15(3), 508-511. doi:10.1590/ S0104-11692007000300023

Sentse, M., Kretschmer, T., \& Salmivalli, N. (2015). The longitudinal interplay between bullying, victimization, and social status: Age-related and gender differences. Social Development, 24(3), 659-677. doi:10.1111/sode. 12115 
Silva, J. L., \& Bazon, M. R. (2014). Educação escolar e conduta infracional em adolescentes: Revisão integrativa da literatura. Estudos de Psicologia (Natal), 19(4), 278-287. doi:10.1590/S1413$-294 X 2014000400005$

Silva, J. L., Oliveira, W. A., Braga, I. F., Farias, M. S., Silva Lizzi, E. A., Gonçalves, M. F. C., ...Silva, M. A. I. (2016). The effects of a skill-based intervention for victims of bullying in Brazil. International Journal of Environmental Research and Public Health, 13, 1042-1052. doi:10.3390/ ijerph13111042

Silva, J. L., Oliveira, W. A., \& Longarezi, A. M. (2008). O bullying e suas interferências nos processos de ensino-aprendizagem. Revista da Sociedade de Psicologia do Triângulo Mineiro, 12(2), 177-185.

Silva, M. A. I., Silva, J. L., Pereira, B. O., Oliveira, W. A., \& Medeiros, M. (2014). The view of teachers on bullying and implications for nursing. Revista da Escola de Enfermagem da USP, 48(4), 723 730. doi:10.1590/S0080-623420140000400021

Skrzypiec, G., Slee, P., Murray-Harvey, R., \& Pereira, B. (2011). School bullying by one or more ways: Does it matter and how do students cope? School Psychology International 32(3), 288 311. doi:10.1177/0143034311402308

Stan, C., \& Beldean, I. G. (2014). The development of social and emotional skills of students - Ways to reduce the frequency of bullying-type events. Experimental results. Procedia - Social and Behavioral Sciences, 114, 735-743. doi:10.1016/j. sbspro.2013.12.777
Thunfors, P., \& Cornell, D. (2008). The popularity of middle school bullies. Journal of School Violence, 7(1), 65-82. doi:10.1300/J202v07n01_05

Ttofi, M. M., \& Farrington, D. P. (2011). Effectiveness of school-based programs to reduce bullying: A systematic and meta-analytic review. Journal of Experimental Criminology, 7(1), 2756. doi:10.1007/s11292-010-9109-1

Viechtbauer, W. (2010). Conducting meta-analyses in $\mathrm{R}$ with the metafor package. Journal of Statistical Software, 36(3), 1-48. doi:10.18637/jss. v036.i03

Wölfer, R., \& Scheithauer, H. (2014). Social influence and bullying behavior: Intervention-based network dynamics of the fairplayer.manual bullying prevention program. Aggressive Behavior, 40(4), 309-331. doi:10.1002/ab.21524

(C) O(s) autor(es), 2018. Acesso aberto. Este artigo está distribuído nos termos da Licença Internacional Creative Commons Atribuição 4.0 (http://creativecommons.org/licenses/by/4.0/), que permite o uso, distribuição e reprodução sem restrições em qualquer meio, desde que você dê crédito apropriado ao(s) autor(es) original(ais) e à fonte, fornecer um link para a licença Creative Commons e indicar se as alterações foram feitas. 